\title{
The effect of ionization on the global minima of small and medium sized silicon and magnesium clusters
}

\author{
Sandip De, ${ }^{1}$ S. Alireza Ghasemi, ${ }^{1}$ Alexander Willand, ${ }^{1}$ Luigi Genovese, ${ }^{2}$ Dilip Kanhere,${ }^{3}$ and Stefan Goedecker ${ }^{1}$ \\ ${ }^{1}$ Department of Physics, Universität Basel, Klingelbergstr. 82, 4056 Basel, Switzerland \\ ${ }^{2}$ European Synchrotron Radiation Facility, 6 rue Horowitz, BP 220, 38043 Grenoble France \\ 3 Department of Physics and Center for Modeling and Simulation, \\ University of Pune, Ganeshkhind, Pune 411 00\%, India
}

(Dated: May 24, 2022)

\begin{abstract}
We reexamine the question of whether the geometrical ground state of neutral and ionized clusters are identical. Using a well defined criterion for being 'identical' together extensive sampling methods on a potential energy surface calculated by density functional theory, we show that the ground states are in general different. This behavior is to be expected whenever there are meta-stable configurations which are close in energy to the ground state, but it disagrees with previous studies.

PACS numbers:
\end{abstract}

\section{INTRODUCTION}

Since experimental mass selection methods require ionized systems, the majority of experimental information on clusters was obtained for ionized clusters. On the other hand, neutral systems are of greater practical interest and the majority of theoretical works are done on neutral systems. The relation between the properties of neutral and ionized clusters is therefore an important one. The basic property which determines all other properties is the structure. Finding the global minimum structure of a cluster is a complex global geometry optimization problem on a high dimensional potential energy landscape [1] with a huge number of local minima. In order to make accurate structural predictions, the potential energy surface should be calculated within density functional theory. Doing exhaustive unbiased searches for the global minimum at the density functional level has only recently become possible through the combined improvements in global optimization algorithms and computer performance.

One basic question concerning the relation between neutral and ionized clusters is whether they have the same basic structure. Evidently adding or removing one electron will change the the exact bond lengths and angles but one might suspect that the structures remains nevertheless very similar. The relation between the structure of neutral and ionized clusters has been investigated in numerous previous publications for the same silicon and magnesium clusters that we have reexamined. The conclusion, in all the publications we are aware of, is that in general the structures of the neutral and cation clusters are more or less identical, but the criteria for being 'identical' are not always explicitly given. We introduce a well defined criterion for being identical. Two minima are identical or more precisely 'related', if the equilibrium structure of the ionized system lies within the catchment basin of the neutral system and vice versa.Applying this criterion on an extensive database of accurately relaxed geometries, we arrive at the opposite conclusion.

\section{METHODOLOGY}

The global and local minima presented here are obtained within Density functional theory using the'Big DFT' wavelet code [2] which was coupled to the 'minima hopping' [3] global optimization algorithm. The local spin density approximation (LDA) is used together with HGH type pseudo potentials [4] for the calculation of the potential energy surface. The size of the wavelet basis set was chosen such that the energies were converged to within better than $10^{-4}$ Hartree with respect to the infinite size basis set. A combination of conjugate gradient and BFGS methods 5] was used for the local geometry optimizations and they were stopped when the numerical noise in the forces was about 20 percent of the total force. This happened usually when the largest force acting on any atom was less than $2 \times 10^{-5}$ Hartree/Bohr. Saddle points were found by a modified version of the 'A spline for your saddle' method [6].

In contrast to plane wave basis sets, free boundary conditions for charged systems are not problematic with a wavelet basis set. In plane wave program a neutralizing background charge is needed, since a periodic system can not have a charged unit cell. In a wavelet basis set the integral equation for the potential $V$

$$
V(\mathbf{r})=\int \frac{\rho\left(\mathbf{r}^{\prime}\right)}{\left|\mathbf{r}-\mathbf{r}^{\prime}\right|} d \mathbf{r}^{\prime}
$$

can be solved directly for the electronic charge density $\rho$ with a monopole and the electrostatic potential can therefore be calculated very accurately for charged systems [7].

For all the clusters we have carried out separate global optimization runs for neutral and ionized system. Since anions with weakly bound additional electrons are less accurately described by density functional theory than cations, we considered only cations in addition to the neutral system. For small clusters ( less than 10 atoms for silicon and less than 20 atoms for magnesium) the majority of low energy local minima can be obtained. That 
this condition is fulfilled can be deduced in the minima hopping algorithm from a strong increase in the kinetic energy of the molecular dynamics trajectories. For larger clusters this explosion of the kinetic energy 8] can not be observed for any reasonable short simulation time. In case of medium sized clusters we calculated always at least 100 low energy local minima structures and we did various empirical checks to convince ourselves that the global minimum was found. We checked for instance always that the lowest energy structures found for the cation system did not relax upon addition of an electron into a structure that was lower in energy than the putative global minimum found for the neutral system.

Using this approach we investigate whether the global minimum structures of neutral and positively charged clusters are related. We will use the following two criteria as the definition for two structures of a neutral and ionized system to be "related"

- The equilibrium structure $i$ of the cation will relax into the equilibrium structure $j$ of the neutral cluster when an electron is added.

- The equilibrium structure $j$ of the neutral cluster will relax into the equilibrium structure $i$ of the cation when an electron is removed.

By relaxations we mean local geometry optimization with a sufficiently small step size, which will make it very unlikely that the local geometry optimization jumps out of the catchment basin within which the local geometry optimization was started. The structures of the neutral and ionized system are thus considered to be related, if there is a one-to-one mapping between the global minima structures upon addition and removal of an electron. This definition of two structures being related is motivated by the fact that the removal or addition of an electron in an experiment is quasi instantaneous on the time scale of the motion of the heavy nuclei. A cluster will therefore relax experimentally into the minimum of the catchment basin in which it finds itself after the addition or removal of an electron. In order to see whether our definition is fulfilled or not, we have introduced mapping charts that show which local minimum of the neutral system relaxes into which local minimum of the ionized system and vice versa. We consider the global minima structures of the neutral and ionized cluster to be identical if the two global minima structures are related according to the above definition.

In order to detect the degree of similarity between two structures with $N_{a t}$ atoms and atomic coordinates $R^{a}$ and $R^{b}$ respectively we have also calculated the configurational distance $D$

$$
D=\frac{1}{N_{a t}} \sqrt{\sum_{i=1}^{3 N_{a t}}\left(\mathbf{R}_{i}^{a}-\mathbf{R}_{i}^{b}\right)^{2}}
$$

The two structure were rotated and shifted in such a way as to minimize $D$. In addition atomic numbers were permutated in the search for the smallest possible $D$. It turns out that structures, that are related according to our definition, usually have also a small configurational distance, but the opposite is not true.

We have chosen silicon and magnesium clusters for this study since they are among the most extensively studied clusters and since we wanted to see whether clusters made out of insulating and metallic materials behave in the same way.

The figures are produced using 'v_sim'(http://inac.cea.fr/L_Sim/V_Sim/index.en.html). The symmetry group was found using vmd [9] plug-ins [10].

\section{RESULTS}

\section{A. Silicon Clusters}

For silicon system we did our calculation for small clusters containing $3-19$ atoms and for $S i_{32}$ as an representative of medium size clusters. For very small clusters there exist only a few local minima structures and they are therefore usually well separated in energy. As the number of atoms in the cluster grows, the number of meta-stable structures increases exponentially. The concept of a global minimum is already rather ill-defined for silicon clusters containing more than some 7 atoms. They have many quite distinct structures that are very close in energy to the global minimum structure [11]. As a consequence more than one structure can be populated even at room temperature. A second consequence of this is that different density functionals can give a different energetic ordering of the various minima 12] and even with the most accurate Quantum Monte Carlo calculations it is difficult to obtain the resolution necessary to predict the correct energetic ordering [11]. In this study we are not claiming to identify the correct ground state structures of the studied silicon clusters, but instead we want to show general trends. Therefore we use standard density functional theory instead of the extremely expensive Quantum Monte Carlo method. Considering the fact that completely different structures can be extremely close in energy suggests strongly that a major perturbation such as the addition or removal of an electron can change the energetic ordering of the structures. Older studies have in contrast frequently just assumed that the ground state structures of neutral and positively charged clusters are the same. In some more recent investigations, few cases were identified where the neutral and positively charged cluster were not 'related'. In an investigation, where silicon clusters with less than 20 atoms were investigated [13], $S i_{8}, S i_{12}, S i_{13}, S i_{15}$ and $S i_{17}$ were found as the exceptions were the ground state geometries of the cation differ from the one of the neutrals. In another investigation of silicon clusters with less than 10 atoms [14], the ground state geometry of $S i_{9}$ and $S i_{10}$ were found to be the "related". Both studies are in contradiction to 


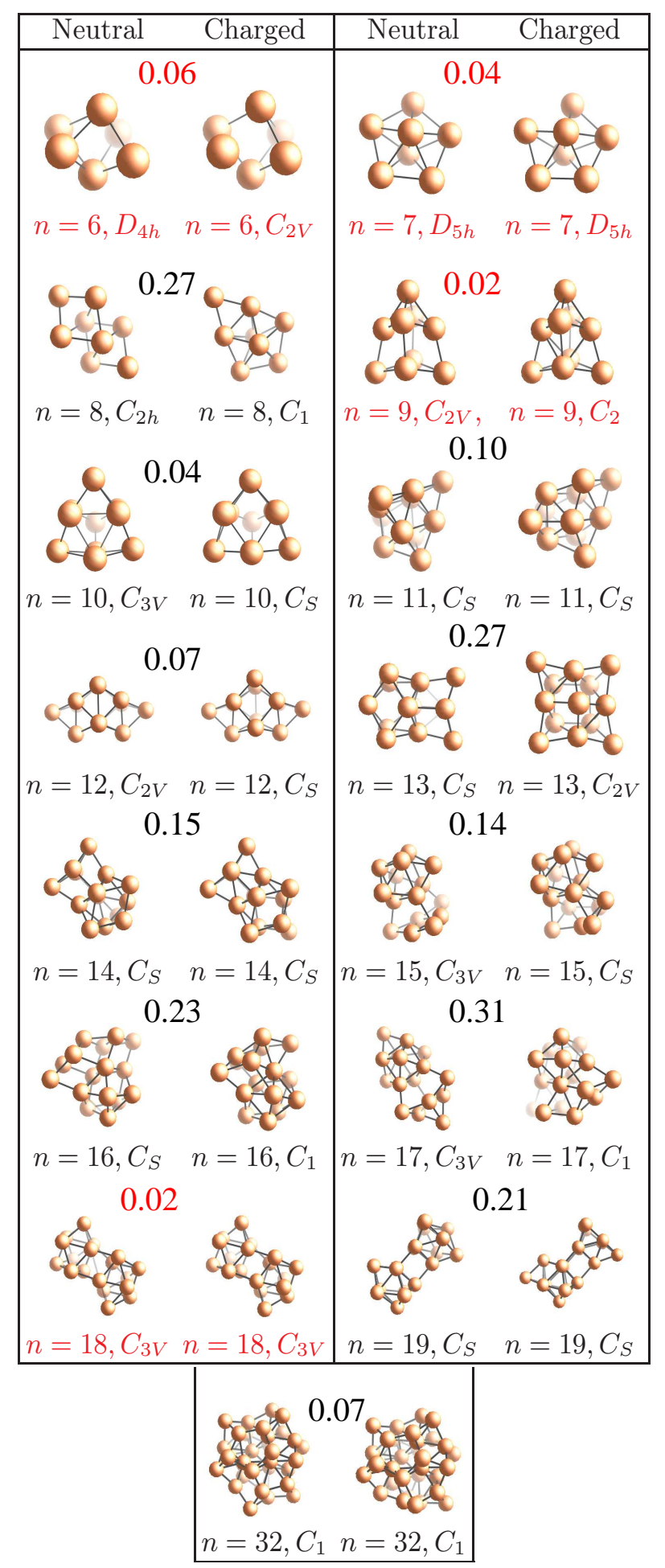

FIG. 1: Global minima of charged and neutral $S i_{n}$, for $\mathrm{n}=6,7, . .19$ and 32 . Only for $\mathrm{n}=6,7,9$ and 18 the global minima of charged and neutral are "related". The configurational distance between each pair is given in $\AA$.

our results which show that for silicon clusters with more than 7 atoms, the ground state structures of the neutrals and cations are not related with the only exception of

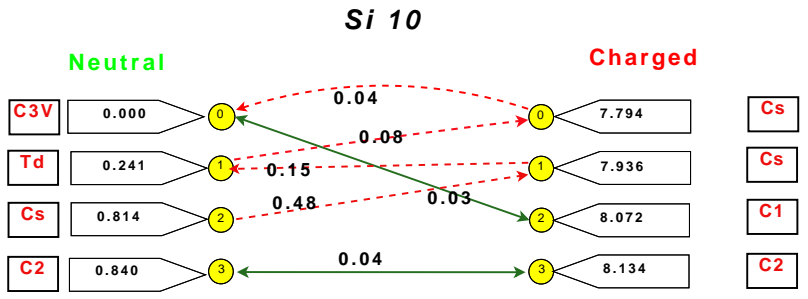

FIG. 2: Mapping chart for $S i_{10}$. The configurational distance between the the neutral and charged ground state configurations is very small $(0.04 \AA)$ and ionized ground state does relax into the neutral ground state when an electron is added. However the neutral ground state does not relax into the ionized ground state and therefore the structures are not 'related' according to our definition. This behavior is rather exceptional and was only found for $S i_{10}, S i_{12}, M g_{25}$ and $M g_{56}$. For all

the other unrelated structures neither the ionized ground state relaxes into the neutral ground state nor the neutral into the ionized one.

\section{Si 14}

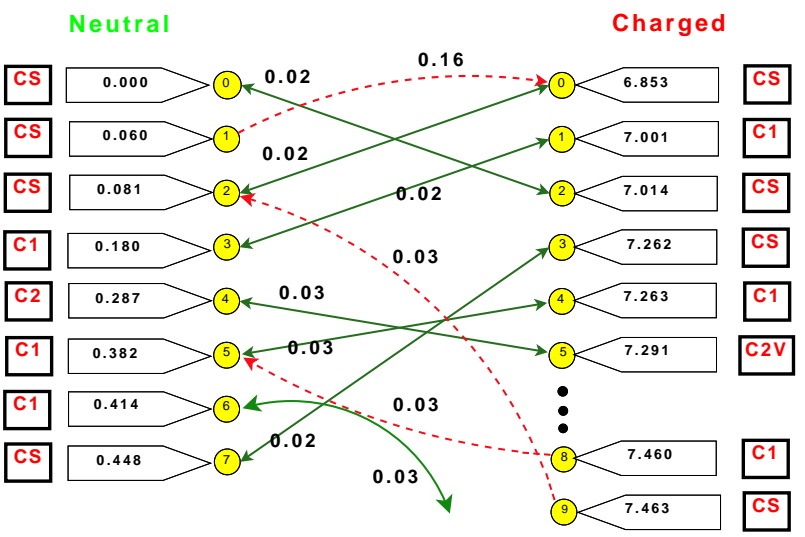

FIG. 3: Mapping chart for $S i_{14}$. The ground state structures are not related and are quite different(FIg: 1).

$S i_{9}$ and $S i_{18}$ and are as a matter of fact quite different( Fig. 11). In another study of medium sized clusters [15] it was also found that in most cases the structures of the neutrals and cations are the same. Out of the medium size clusters we have only examined the 32 atom cluster for which we however also find different ground state structures.

Fig: 2 and Fig: 3 shows the mapping chart which gives detailed information about the relaxation properties upon addition and removal of an electron. We distinguish between reversible and irreversible mappings between pairs of local minima. The energies of all the structures are measured with respect to the ground state energy of the neutral system. Solid double arrow connecting lines denote reversible mappings and dashed single arrow connecting line irreversible mappings. The space group is given in the rectangular boxes and the numbers close to the the connecting lines give the configurational 
distance of the two configurations. A reversible mapping connects two structures which are related according to our definition. In an irreversible mapping, the cluster relaxes from the $\mathrm{i}$-th to the $\mathrm{j}$-th local minimum when an electron is removed or added, but it relaxes to a structure which is different form the i-th when the electron is given back or taken away again. Fig: 2 and Fig: 3 shows that both kinds of mappings are encountered frequently. The minima of the neutral and cation are related according to our aforementioned definition only if a reversible mapping connects the two global minima. This case was never encountered for clusters of more than 7 atoms except for $S i_{9}$ and $S i_{18}$ and the global minimum structures for the neutrals and cations are thus different except for $S i_{n}$ n=3 to 7,9 and 18 in this size range. The numerical values along the relaxation arrows in the mapping diagrams indicate the configurational distances in the relaxation processes. These distances are typically of the order of $0.03 \AA$, and thus show that the distortion during the relaxation is rather small. The symmetry group is also conserved in most cases. The fact that the geometries change so little upon removal or addition of an electron might have contributed to the wrong believe that the ground state of the neutral and cation are more or less identical. Nevertheless these small displacements are frequently sufficient to bring the system in another catchment basin.

The energetic ordering for neutral and ionized cluster configurations would be identical if the ionization energy or electron affinities (including the energy that comes from the small relaxation upon removal or addition of an electron) would be constant, i.e. independent of the shape of the various meta-stable configurations. The essential point is however that ionization energies and electron affinities are about two orders of magnitude larger than the energy differences between the ground state structure and the next meta-stable low energy structures. Relatively small differences in the ionization energies and electron affinities between the different configurations can therefore lead to a reversal of the energetic ordering of the local minima. The energy differences between the ground state and the first meta-stable configuration is of the order of few $k_{B} T$ at room temperature and the energy differences between the higher meta-stable configurations are even smaller.

We find small configurational distance values not only for the structural changes induced by the addition or removal of an electron but also between different local minima of the neutral and ionized clusters. The configurational distance between the first and second meta-stable configuration of the $S i_{14}$ cluster is for instance only 0.15 $\AA$. Nevertheless the two local minima are separated by a barriers of about $1.2 \mathrm{eV}$. In these disordered structure a broad distribution of barrier heights is to be expected [16] and we find indeed also low barriers. The configurational distance between the ground state of the charged $S i_{10}$ cluster and its first meta-stable configuration is for instance also $0.15 \AA$. But the barrier between the two structures are much smaller namely $0.22 \mathrm{eV}$ and $0.08 \mathrm{eV}$ respectively. Such small barrier heights are well below the accuracy level of density functional methods and it can hence not be excluded that higher level calculations such as coupled cluster or Quantum Monte Carlo calculation would give a different potential energy surface. Our previous experience [17] shows however that barrier height are quite well reproduced by density functional theory if no bonds are broken during the transformation from one structure to the other.

\section{B. Magnesium clusters}

For $M g_{n}$ we have systematically studied all small and medium size clusters with $\mathrm{n}=6$ to 30 atoms as well as $M g_{56}$. The Global minima are shown in Fig. 4 and Fig. 5 . For these cluster sizes the electronic HOMO-LUMO gap does not yet tend to zero, but is around $0.1 \mathrm{eV}$. So no pronounced metallic behavior is present. The ionization energies are also comparable to the case of the silicon clusters. The ionization energy is on average $5 \mathrm{eV}$ for the magnesium clusters and $7 \mathrm{eV}$ for the silicon clusters. The only notable difference we found between the silicon and magnesium cluster is number of meta-stable states, which is much larger for silicon clusters. Since all energy differences are however also smaller for $M g$ than for $S i$ ,the average configurational distance between different meta-stable configurations is again similar in both cases. Hence $M g$ clusters have the same overall behavior as the $S i$ clusters, i.e in general the neutral and ionized ground states are not related.

In the studied size range we find that the global minima of neutral and cation clusters are related for $\mathrm{n}=7,8,12,15,17,18,19,24,26,27,30$ and 32 . For a bigger system , $M g_{56}$ we also found the global minima to be different for charged and neutral system. So in total the ground state structures are related in 12 cases and unrelated in 21 cases. Fig. 6] and Fig. 7 exemplifies the same kind of mapping for $M g_{16}$ and $M g_{24}$ between charged and neutral system as we already showed for silicon systems. These mapping charts look very similar to that of silicon systems, i.e. the energetic ordering changes when the system goes from the neutral to the charged state. Although for $\mathrm{Mg}_{24}$ the neutral and charged global minima are 'related', from the mapping chart (Fig. (7) we can see the sign of energetic ordering changes in the system while going from neutral to charged state. The numerical values along the relaxation arrows in the mapping diagrams indicate the configurational distances in the relaxation processes. These distances are typically of the order of $0.02 \AA$, unlike Silicon systems where this value is $0.03 \AA$, and thus show that the distortion during the relaxation is smaller than that of silicon systems.

Our results are again overall in disagreement with the majority of previous publications. In one of the earliest publication on this topic, where clusters with up to 6 atoms were studied, identical ground state structures 


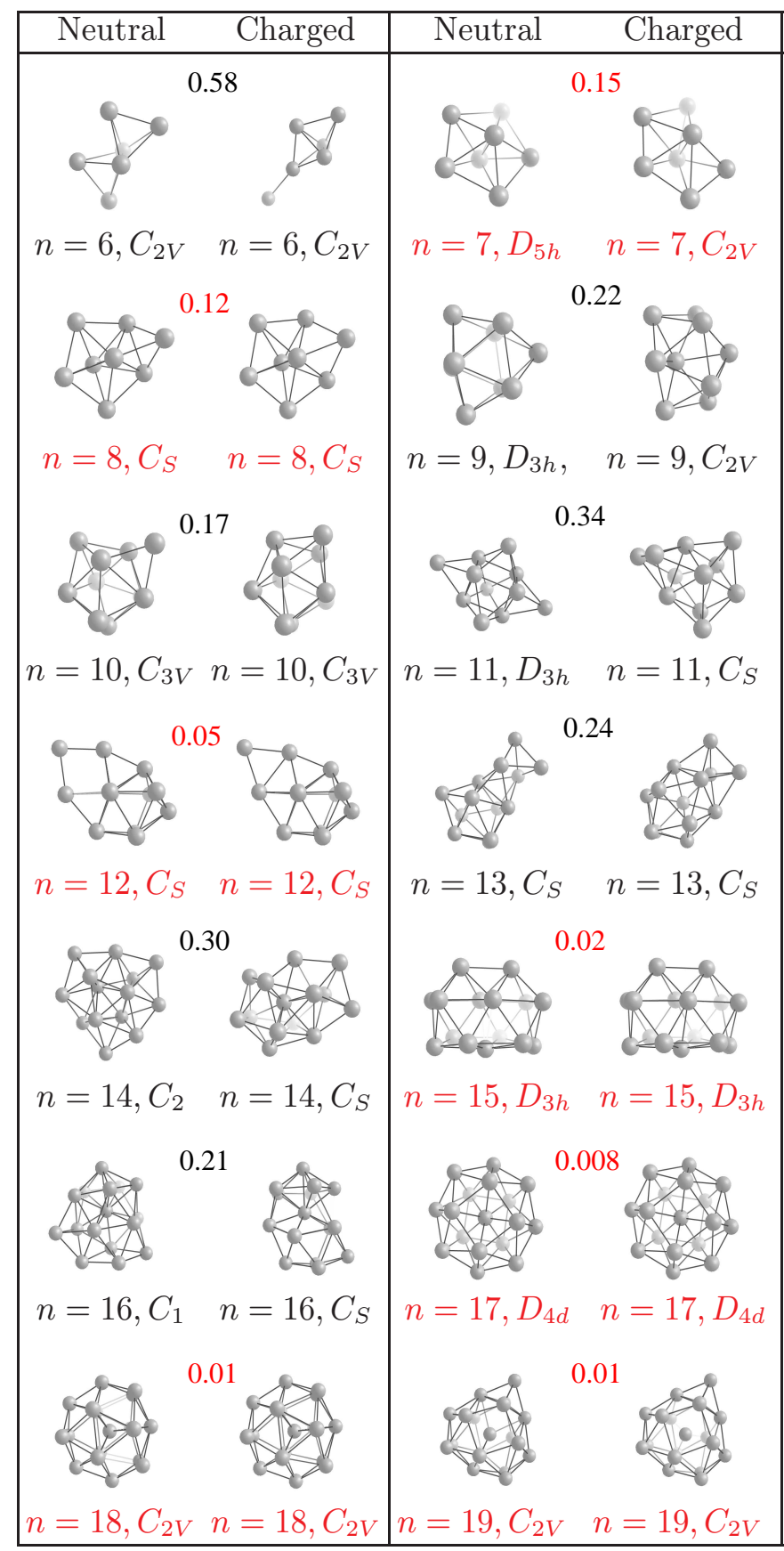

FIG. 4: Global minima of charged and neutral $M g_{n}$, for $\mathrm{n}=6$-19. Only for $\mathrm{n}=7,8,12,15,17,18$ and 19 the global minima of charged and neutral are "related". The configurational distance between each pair is given in $\AA$.

were found for $M g_{6}$ and $M g_{7}$ [18]. In a study of Mg cluster with up to 21 atoms, it was found that only for $M g_{3}$ and $\mathrm{Mg}_{4}$ the ground states are different [19]. In another somewhat more extensive study in the range between 2 and 22 atoms 20], it was found that in addition also $M g_{6}, M g_{7}, M g_{8}, M g_{11}, M g_{12}$ and $M g_{13}$ have different ground states.

We have also recalculated the energetic ordering of the

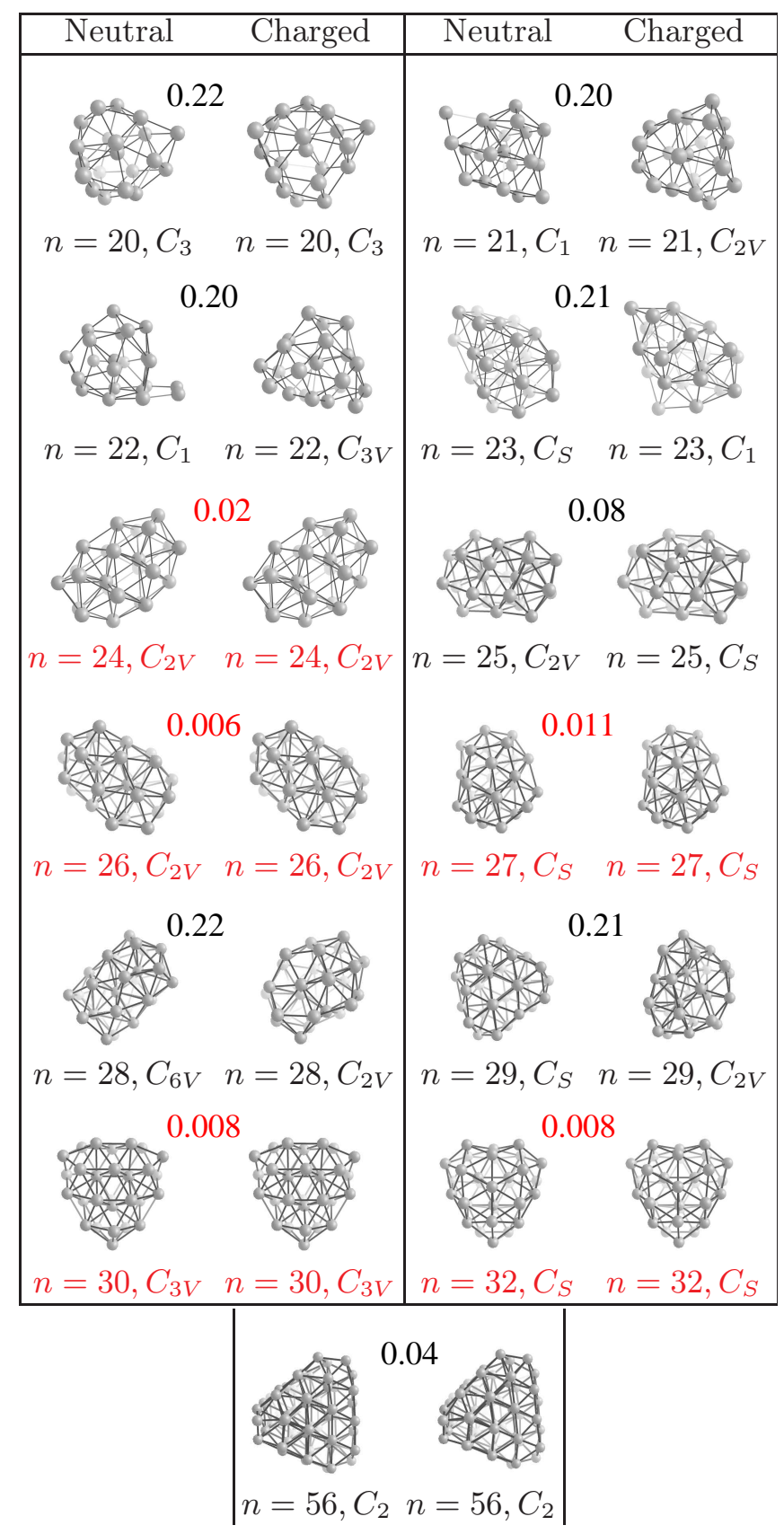

FIG. 5: Global minima of charged and neutral $M g_{n}$, for $n=20-30,32,56$. Only for $n=24,26,27,30$ and 32 the global minima of charged and neutral are "related". The configurational distance between each pair is given in $\AA$

minima of several magnesium clusters with the PBE functional [21]. In all these cases the ordering was identical to the ordering with the LSD functional. This is in contrast to the silicon clusters where the energetic ordering depends on the functional being used. This suggests that the density functional results for the magnesium clusters are very reliable.

For the magnesium clusters the average configurational distance between the various local minima is typically in 


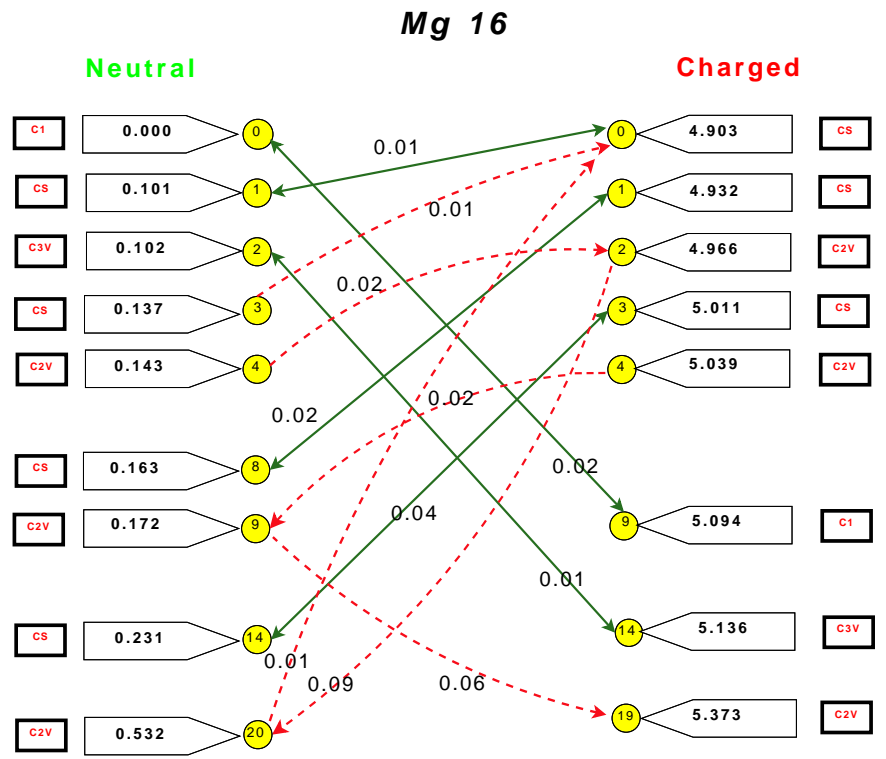

FIG. 6: Mapping chart for $M g_{16}$. The ground state of the neutral cluster is mapped to a rather high local minimum of the charged cluster.

\section{Mg 24}

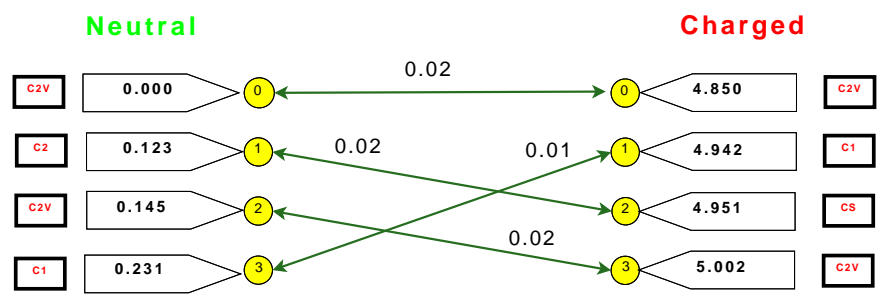

FIG. 7: Mapping chart for $M g_{24}$. For this system the ground states are related. The higher energy meta-stable states are however even for such a system typically not 'related'.

the range between $0.1 \AA$, and $0.2 \AA$, and thus larger than the average configurational distance of the relaxation induced the the removal or addition of electrons. Since the magnesium cluster are also disordered we find, as in the case of the silicon clusters, a broad distribution of barrier heights. We calculated randomly 12 barrier heights of the neutral $M g_{16}$ and $M g_{24}$ cluster and we found values in between 0.05 and $0.8 \mathrm{eV}$.

\section{CONCLUSION}

Using an exhaustive sampling of the low energy configurations based on the minima hopping method we show for silicon and magnesium clusters that the ground states of neutral and ionized clusters are in general not related and are in many cases quite different. This comes from the fact that for medium and large clusters there are in general numerous meta-stable structures which are energetically very close to the ground state. The differences in ionization energies and electron affinities for different structures are much larger than this energy difference between structures. These facts have to be taken into account in the interpretation of experiments with ionized clusters.

There is no reason to believe that clusters made out of other elements behave differently. Based on our arguments one can only expect that for certain magic cluster sizes, for which ground state structures exist that are considerably lower in energy than other competing metastable structures, the ground state does not change upon removal or addition of an electron. Such an example is for instance the $C_{60}$ fullerene.

\section{ACKNOWLEDGMENT}

We thank the Indo-Swiss Research grant and SNF for the financial support and the CSCS for computer facility.
[1] David wales. Energy Landscapes: Applications to Clusters, Biomolecules and Glasses (Cambridge Molecular Science). Cambridge University press, 2003.

[2] Luigi Genovese, Alexey Neelov, Stefan Goedecker, Thierry Deutsch, Seyed Alireza Ghasemi, Alexander Willand, Damien Caliste, Oded Zilberberg, Mark Rayson, Anders Bergman, and Reinhold Schneider. Daubechies wavelets as a basis set for density functional pseudopotential calculations. The Journal of Chemical Physics, 129(1):014109, 2008.

[3] Stefan Goedecker. Minima hopping: An efficient search method for the global minimum of the potential energy surface of complex molecular systems. The Journal of Chemical Physics, 120(21):9911-9917, 2004.

[4] C. Hartwigsen, S. Goedecker, and J. Hutter. Relativistic separable dual-space gaussian pseudopotentials from $\mathrm{h}$ to rn. Phys. Rev. B, 58(7):3641-3662, Aug 1998.

[5] Jorge Nocedal. Updating quasi-newton matrices with limited storage. Mathematics of Computation, 35(151):pp. 773-782, 1980.

[6] Rebecca Granot and Roi Baer. A spline for your saddle. The Journal of Chemical Physics, 128(18):184111, 2008.

[7] Luigi Genovese, Thierry Deutsch, Alexey Neelov, Stefan Goedecker, and Gregory Beylkin. Efficient solution of poisson's equation with free boundary conditions. The Journal of Chemical Physics, 125(7):074105, 2006.

[8] Stefan Goedecker. published in Modern Methods of Crystal Structure Prediction, Edited by Artem R. Oganov. Wiley-VCH, 2011.

[9] William Humphrey, Andrew Dalke, and Klaus Schulten. VMD - Visual Molecular Dynamics. Journal of Molecular Graphics, 14:33-38, 1996. 
[10] John Stone, Justin Gullingsrud, Paul Grayson, and Klaus Schulten. A system for interactive molecular dynamics simulation. In John F. Hughes and Carlo H. Séquin, editors, 2001 ACM Symposium on Interactive 3D Graphics, pages 191-194, New York, 2001. ACM SIGGRAPH.

[11] Waldemar Hellmann, R. G. Hennig, Stefan Goedecker, C. J. Umrigar, Bernard Delley, and T. Lenosky. Questioning the existence of a unique ground-state structure for si clusters. Phys. Rev. B, 75(8):085411, Feb 2007.

[12] Soohaeng Yoo and X. C. Zeng. Structures and relative stability of medium-sized silicon clusters. iv. motif-based low-lying clusters si[sub 21]-si[sub 30]. The Journal of Chemical Physics, 124(5):054304, 2006.

[13] Liu Bei. ; Lu Zhong-Yi ; Pan Bicai ;Wang Cai-Zhuang ; Ho Kai-Ming ; Shvartsburg Alexandre A ; Jarrold Martin F. Ionization of medium-sized silicon clusters and the geometries of the cations. Jcp, 109(21):9401-9409, 1998.

[14] Xu-yan Zhou Li Bao-xing, Pei-lin Cao. Electronic and geometric structures of $\sin$ and $\sin +(\mathrm{n}=210)$. phys. stat. sol. (b), 2003.

[15] R. L. Zhou and B. C. Pan. Low-lying isomers of si[sub $\mathrm{n}][$ sup + $]$ and si[sub n] $[$ sup -] $(\mathrm{n}=31-50)$ clusters. The Journal of Chemical Physics, 128(23):234302, 2008.
[16] A. Heuer, Journal of Physics: Condensed Matter 20, 373101 (2008) ; G. Daldoss, O. Pilla, G. Viliani, C. Brangian and G. Ruocco, Phys. Rev. B 603200 (1999)

[17] S. Alireza Ghasemi, Maximilian Amsler, Richard G. Hennig, Shantanu Roy, Stefan Goedecker, C. J. Umrigar, Luigi Genovese, Thomas J. Lenosky, Tetsuya Morishita, Kengo Nishio: Phys. Rev. B 81, 214107 (2010)

[18] F. Reuse, S. N. Khanna, V. de Coulon, and J. Buttet. Behavior of magnesium clusters under electron attachment and detachment. Phys. Rev. B, 39(17):12911-12914, Jun 1989.

[19] Andrey Lyalin, Ilia A. Solov'yov, Andrey V. Solov'yov, and Walter Greiner. Evolution of the electronic and ionic structure of $\mathrm{mg}$ clusters with increase in cluster size. Phys. Rev. A, 67(6):063203, Jun 2003.

[20] Julius Jellinek and Paulo H. Acioli. Magnesium clusters: structural and electronic properties and the size-induced nonmetal-to-metal transition. The Journal of Physical Chemistry A, 106(45):10919-10925, 2002.

[21] John P. Perdew, Kieron Burke, and Matthias Ernzerhof. Generalized gradient approximation made simple. Phys. Rev. Lett., 77(18):3865-3868, Oct 1996. 